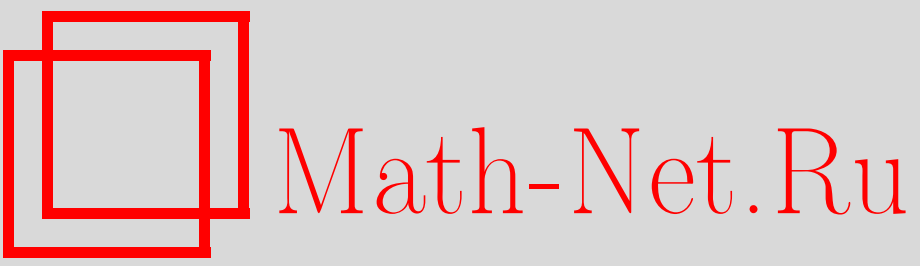

В. В. Пупышев, Ложные слагаемые в задаче трех частиц, ТМФ, 2000, том 125, номер 2, 253-271

DOI: https://doi.org/10.4213/tmf667

Использование Общероссийского математического портала Math-Net.Ru подразумевает, что вы прочитали и согласны с пользовательским соглашением

http://www.mathnet.ru/rus/agreement

Параметры загрузки:

IP : 54.224 .60 .19

26 апреля 2023 г., $16: 21: 53$ 
ТЕОРЕТИЧЕСКАЯ

И МАТЕМАТИЧЕСКАЯ

ФИЗИКА

Том 125, № 2

ноябрь, 2000

(C) 2000 г.

В. В. Пупышев*

\section{ЛОЖНЫЕ СЛАГАЕМЫЕ В ЗАДАЧЕ ТРЕХ ЧАСТИЦ}

Метод гипергармоник применяется для построения разбиений центральных парных взаимодействий и фаддеевских компонент волновой функции трехчастичной системы на физические и ложные слагаемые. Сумма физических слагаемых взаимодействий или фаддеевских компонент по всем парам частиц отлична от нуля, в то время как суммы ложных слагаемых и взаимодействий, и фаддеевских компонент по всем парам частиц тождественно равны нулю. Доказан критерий существования ложных слагаемых. Показано, что достаточное условие этого критерия равносильно закону сохранения некоторого квантового числа.

\section{1. ВВЕДЕНИЕ}

Понятие о полном наборе сохраняющихся квантовых чисел является одним из основных в квантовой механике [1]. Полный набор состоит из физических величин, обладающих следующими свойствами. Во-первых, эти величины измеримы одновременно. Во-вторых, если они одновременно имеют определенные значения в некотором состоянии, то любая другая физическая величина, не являющаяся их функцией, не может иметь определенного значения в этом же состоянии. Полное описание состояния невозможно без знания полного набора его квантовых чисел, поэтому построение такого набора является одной из основных задач квантовой механики.

В настоящей работе мы исследуем задачу такого рода для системы трех бесспиновых нетождественных частиц с произвольными массами $m_{1}, m_{2}, m_{3}$ и центральными парными взаимодействиями $V_{k}$, где $k=1,2,3$ - номер пары частиц с номерами $i$ и $j$. Наша главная цель - доказать, что полный набор сохраняющихся квантовых чисел любого состояния такой системы содержит особое квантовое число $p_{u}=1$, если каждое парное взаимодействие есть сумма

$$
V_{k}=V_{k}^{u}+V_{k}^{s}
$$

слагаемых двух разных типов, а именно таких, что сумма слагаемых первого типа по всем парам частиц не равна тождественно нулю, а сумма слагаемых второго типа по

* Объединенный институт ядерных исследований, Дубна, Московская обл., Россия. E-mail: pupyshev@thsun1.jinr.ru 
всем парам частиц тождественно равна нулю:

$$
\sum_{k=1}^{3} V_{k}^{u} \neq 0, \quad \sum_{k=1}^{3} V_{k}^{s} \equiv 0
$$

Слагаемые $V_{k}^{u}$ и $V_{k}^{s}$ будем называть физическими и ложными, соответственно, потому что первые содержатся в полном взаимодействии $V$, а вторые не дают в него никакого вклада (взаимно компенсируют друг друга при суммировании по всем парам частиш):

$$
V=\sum_{k=1}^{3} V_{k}=\sum_{k=1}^{3} V_{k}^{u}
$$

Пусть $H_{0}$ и $E$ - свободный гамильтониан и полная энергия рассматриваемой трехчастичной системы, а $\Psi$ - волновая функция некоторого состояния такой системы. Уравнение Шредингера [1] для функции $\Psi$ содержит парные взаимодействия только в виде их суммы $V$, а каждое из трех $(i=1,2,3)$ уравнений Фаддеева [2] для фаддеевских компонент $\Psi_{i}$ этой же функции

$$
\Psi=\sum_{k=1}^{3} \Psi_{k}
$$

содержит соответствующее парное взаимодействие $V_{i}$. Поэтому для взаимодействия $(1),(2)$ ложные слагаемые отсутствуют в уравнении Шредингера:

$$
\left(H_{0}-E\right) \Psi=-V \Psi=-\left(\sum_{k=1}^{3} V_{k}^{u}\right) \Psi
$$

но содержатся в уравнениях Фаддеева наряду с физическими:

$$
\left(H_{0}-E\right) \Psi_{i}=-V_{i} \Psi=-\left(V_{i}^{u}+V_{i}^{s}\right) \sum_{k=1}^{3} \Psi_{k}, \quad i=1,2,3
$$

поэтому возникает вопрос об эквивалентности уравнения Шредингера (4) и уравнений Фаддеева (5).

Ложные слагаемые нетрудно построить в частном случае, когда все три частицы тождественные бозоны. Пусть $I$ - единичный оператор, а $S^{+}$- оператор симметризации по всем перестановкам таких бозонов. В рассматриваемом случае парные центральные взаимодействия функционально одинаковые, поэтому $V$ - полностью симметричная функция:

$$
S^{+} V=V=\sum_{k=1}^{3} S^{+} V_{k}, \quad\left(I-S^{+}\right) V=\sum_{k=1}^{3}\left(I-S^{+}\right) V_{k} \equiv 0 .
$$

Следовательно, для каждого парного взаимодействия $V_{k}$ имеет место разбиение $(1)$ на физическое и ложное слагаемые

$$
V_{k}^{u}=S^{+} V_{k}, \quad V_{k}^{s}=\left(I-S^{+}\right) V_{k}, \quad k=1,2,3 .
$$


Физические слагаемые симметричны по всем перестановкам бозонов функции, ложные слагаемые такой симметрией не обладают.

В силу соотношений (6) обшее решение уравнения Шредингера (4) есть сумма двух слагаемых:

$$
\Psi \equiv U+S, \quad U \equiv S^{+} \Psi, \quad S \equiv\left(I-S^{+}\right) \Psi .
$$

Функцию $S$, обладаюшую запрешенной принципом Паули симметрией $\left(S^{+} S \neq S\right)$, часто называют ду́ховым или ложным, т.е. физически нереализуемым, решением уравнения Шредингера (4), а функцию $U$ с разрешенной симметрией $\left(S^{+} U=U\right)$ - физическим решением.

Так как ложные слагаемые $\left(I-S^{+}\right) V_{k}, k=1,2,3$, содержатся в уравнениях Фаддеева (5), то компоненты $\Psi_{1}, \Psi_{2}$ и $\Psi_{3}$, вообше говоря, не являются полностью симметричными: $S^{+} \Psi_{k} \neq \Psi_{k}$. Значит, они представимы в виде разбиений на физические и ложные слагаемые $U_{k}$ и $S_{k}$ :

$$
\Psi_{k}=U_{k}+S_{k}, \quad U_{k} \equiv S^{+} \Psi_{k}, \quad S_{k} \equiv\left(I-S^{+}\right) \Psi_{k} .
$$

При суммировании по всем парам частиц слагаемые $U_{k}$ определяют физическую волновую функцию $U$ с разрешенной симметрией, а слагаемые $S_{k}$ порождают функцию $S$, запрешенную принципом Паули:

$$
U=\sum_{k=1}^{3} U_{k}, \quad S=\sum_{k=1}^{3} S_{k} .
$$

Ложные (запрещенные принципом Паули) слагаемые компонент многочастичной волновой функции неоднократно исследовались [3-9] при анализе различных уравнений для систем, содержаших тождественные частицы. Бенц [3] и Редиш [4] построили такие компоненты при редукции задачи рассеяния для тождественных частиц к системе связанных каналов. Кукулин и Померанцев [5, 6], предложившие и математически обосновавшие метод ортогонального проецирования для итерационного решения многочастичных уравнений в импульсном пространстве, доказали, что расходимость борновских рядов в трехчастичном рассеянии тесно связана с принципом Паули и наличием ду́ховых состояний, запрещенных этим принципом.

Слагаемые фаддеевских компонент, взаимно компенсируюшие друг друга при суммировании по всем парам частищ, Фраер, Гибсон и Пейн [7] назвали ложными. Эти авторы нашли такие слагаемые в явном виде для системы тождественных бозонов с осцилляторными взаимодействиями. Фабр и Ларсен [8] на примере системы нескольких тождественных бозонов в основном состоянии показали, что ложные в аналогичном смысле слагаемые парных потенциалов необходимо исключать, чтобы правильно решать уравнения фаддеевского типа для двухчастичных амплитуд, описывающие лишь двухчастичные корреляции. Необходимость и различные способы исключения ложных (взаимно компенсируюших друг друга) слагаемых из уравнений Фаддеева в конфигурационном пространстве обсуждались в обзоре [9] на примере системы трех тождественных бозонов. 
В настояшей работе строятся и исследуются ложные слагаемые парных взаимодействий и фаддеевских компонент для систем из трех нетождественных частиц и доказывается, что при определенных соотношениях между массами и параметрами парных взаимодействий физические волновые функции подчиняются более общей симметрии, чем перестановочная.

В системе трех тождественных бозонов ложные слагаемые возникают естественным образом как следствие полной перестановочной симметрии полного взаимодействия $V$. Его разложение в ряд по базису из трехчастичных гипергармоник содержит только полностью симметричные базисные функции. Поэтому уравнение Шредингера имеет симметричные относительно любых перестановок физические решения. Гиперсферические разложения отдельных компонент $V_{1}, V_{2}$ и $V_{3}$ полного взаимодействия содержат базисные функции и всех остальных перестановочных симметрий. Следовательно, в уравнениях Фаддеева, содержащих именно эти отдельные компоненты, возникают ложные слагаемые $S_{1}, S_{2}$ и $S_{3}$, обладающие запрешенной принципом Паули симметрией.

Причина появления ложных слагаемых для нетождественных частиц та же, что и в рассмотренном выше случае трех тождественных бозонов: гиперсферические ряды парных взаимодействий содержат, вообе говоря, все базисные гипергармоники, а гиперсферическое разложение полного взаимодействия при определенных условиях на массы частиц и параметры парных взаимодействий не содержит гипергармоник с определенными квантовыми числами. Подсуммы гиперсферических рядов для парных взаимодействий по таким (запрешенным и кинематикой, и формой парных потенциалов) гипергармоникам и будут ложными слагаемыми этих взаимодействий, а оставшиеся подсуммы - физическими.

\section{2. КЛЮЧЕВЫЕ ПОНЯТИЯ И ФОРМУЛЫ}

Напомним понятия теории Фаддеева [2] и метода гипергармоник [10, 11], необходимые для нашего анализа гиперсферических рядов.

Пусть $\left(\mathbf{x}_{i}, \mathbf{y}_{i}\right), i=1,2,3,-$ три набора стандартных приведенных векторов Якоби [2] в шестимерном координатном пространстве $\mathcal{R}^{6}=\mathcal{R}_{x}^{3} \oplus \mathcal{R}_{y}^{3}$ трех рассматриваемых частиц. Каждой паре $\left(\mathbf{x}_{i}, \mathbf{y}_{i}\right)$ сопоставляется шестимерный вектор $\mathbf{r}_{i} \equiv\left(\mathbf{x}_{i}, \mathbf{y}_{i}\right) \in \mathcal{R}^{6}$ с обычными гиперсферическими координатами $\left(r, \Omega_{i}\right)$ [12], где $r \equiv\left(x_{i}^{2}+y_{i}^{2}\right)^{1 / 2}$ - гиперрадиус, а $\Omega_{i} \equiv\left(\hat{x}_{i}, \hat{y}_{i}, \varphi_{i}\right)$ - совокупность пяти гиперсферических углов таких, что $\hat{q}-$ пара сфферических углов трехмерного вектора $\mathbf{q}=\left(\mathbf{x}_{i}, \mathbf{y}_{i}\right), \operatorname{a} \varphi_{i} \equiv \operatorname{arctg}\left(y_{i} / x_{i}\right)$.

В случае центральных парных взаимодействий сохраняются полная энергия $E$, квадрат $\ell(\ell+1)$ полного углового момента $\left(\mathbf{l} \equiv \mathbf{l}_{x_{i}}+\mathbf{l}_{y_{i}}\right)$, его третья проекция $m$ и четность $\sigma$ по отношению к инверсии $\mathbf{r}_{i} \rightarrow-\mathbf{r}_{i}$.

Обычное ограничение, накладываемое на гладкость искомых решений уравнений Шредингера или Фаддеева, - непрерывность всех производных второго порядка во всем пространстве $\mathcal{R}^{6}$.

Пусть $\mathcal{A}^{\varepsilon}$ - класс функций, обладающих указанной гладкостью и заданным набором $\varepsilon \equiv(E, \ell, m, \sigma)$ сохраняюшихся квантовых чисел. В классе $\mathcal{A}^{\varepsilon}$ полный и ортонормированный угловой базис на единичной сфере $\mathcal{S}^{5}$ в $\mathcal{R}^{6}[12]$ образуют вполне определенные 
трехчастичные гипергармоники [11]

$$
Y_{L a b}^{\ell m}\left(\Omega_{i}\right) \equiv N_{L a b}\left(\sin \varphi_{i}\right)^{a}\left(\cos \varphi_{i}\right)^{b} P_{n}^{\left(a+\frac{1}{2}, b+\frac{1}{2}\right)}\left(\cos 2 \varphi_{i}\right) \mathcal{Y}_{a b}^{\ell m}\left(\hat{y}_{i}, \hat{x}_{i}\right)
$$

где

$$
L=a+b+2 n, \quad n=0,1, \ldots, \quad \mathbf{a}+\mathbf{b}=1, \quad(-1)^{a+b}=\sigma .
$$

Гипергармоники аргументов $\Omega_{k}$ и $\Omega_{i}$ связаны унитарным преобразованием, сохраняюшим все квантовые числа набора $\varepsilon$ :

$$
Y_{L c d}^{\ell m}\left(\Omega_{k}\left(\Omega_{i} ; \gamma_{k i}\right)\right)=\sum_{a, b}\left\langle a b\left|K\left(\gamma_{k i}\right)\right| c d\right\rangle_{L \ell} Y_{L a b}^{\ell m}\left(\Omega_{i}\right)
$$

Здесь и всюду далее индексы $a$ и $b$ пробегают все допустимые при заданных $L, \ell$ и $\sigma$ значения, а матричные элементы

$$
\left\langle a b\left|K\left(\gamma_{k i}\right)\right| c d\right\rangle_{L \ell} \equiv\left\langle Y_{L a b}^{\ell m}\left(\Omega_{i}\right) \mid Y_{L c d}^{\ell m}\left(\Omega_{k}\right)\right\rangle \equiv \int_{\mathcal{S}^{5}} d \Omega_{i}\left(Y_{L a b}^{\ell m}\left(\Omega_{i}\right)\right)^{*} Y_{L c d}^{\ell m}\left(\Omega_{k}\right)
$$

оператора кинематического преобразования $K\left(\gamma_{k i}\right)[9,13]$ (коэффициенты Рейналя-Реваи [14]) параметрически зависят от кинематического угла [11]

$$
\gamma_{k i} \equiv g_{k i} \operatorname{arctg}\left(\frac{m_{j}\left(m_{1}+m_{2}+m_{3}\right)}{m_{k} m_{i}}\right)^{\frac{1}{2}}
$$

Если $(k, i)=(1,2),(3,1),(2,3)$, то по определению кинематических углов

$$
g_{k i}=-g_{i k}=1, \quad 0<\gamma_{k i}<\frac{\pi}{2}, \quad \sum_{(k, i)} \gamma_{k i}=\pi .
$$

\section{3. ЛОЖНЫЕ СЛАГАЕМЫЕ ПАРНЫХ ВЗАИМОДЕЙСТВИЙ}

Дадим полное определение физических и ложных слагаемых. Для этого поясним, в каком смысле следует понимать соотношения (2).

Все физические свойства квантового состояния рассматриваемой трехчастичной системы не зависят от математического формализма, используемого для их описания, в частности от того, в каком из трех координатных представлений $\left\langle\mathbf{r}_{i}\right|, i=1,2,3$, записьваются волновая функция $\Psi$ этого состояния и уравнение Шредингера (4), которому она подчинена. Поэтому понятие физических $V_{k}^{u}$ и ложных $V_{k}^{s}$ слагаемых взаимодействия $V_{k}$ не должно зависеть от выбора координатного представления, в котором записываются соотношения (2). В квантовой механике взаимодействие $V_{k}$, а значит, и компоненты $V_{k}^{u}$ и $V_{k}^{s}$ его разбиения (1) всегда понимаются как операторы, а любой оператор всегда определяется в некотором пространстве или же классе функций [1]. Всем упомянутым выше принципам удовлетворяет следуюшее определение физических и ложных слагаемых парных взаимодействий. 
ОПРЕДЕлЕниЕ. Для рассматриваемого класса функций операторы $V_{k}^{u}$ и $V_{k}^{s}$ будем называть физическим и ложным слагаемыми взаимодействия $V_{k}$, если соотношения (2) выполняются в любом из трех координатных представлений $\left\langle\mathbf{r}_{i}\right|, i=1,2,3$, и для любой функции $\Psi$ этого класса:

$$
\begin{aligned}
& \left\langle\mathbf{r}_{i}\left|\sum_{k=1}^{3} V_{k}^{u}\left(\mathbf{r}_{k}\right)\right| \Psi\left(\mathbf{r}_{i}\right)\right\rangle \neq 0, \\
& \left\langle\mathbf{r}_{i}\left|\sum_{k=1}^{3} V_{k}^{s}\left(\mathbf{r}_{k}\right)\right| \Psi\left(\mathbf{r}_{i}\right)\right\rangle \equiv 0 .
\end{aligned}
$$

Выведем достаточные условия существования ложных слагаемых центральных взаимодействий для функций класса $\mathcal{A}^{\varepsilon}$. Парные взаимодействия представим операторными рядами по их собственным угловым базисам (7):

$$
V_{k}\left(x_{k}\right)=\sum_{L, L^{\prime}} \sum_{a, b}\left|Y_{L a b}^{\ell m}\left(\Omega_{k}\right)\right\rangle V_{k a b}^{L L^{\prime}}(r)\left\langle Y_{L^{\prime} a b}^{\ell m}\left(\Omega_{k}\right)\right|, \quad k=1,2,3 .
$$

Использовав эти ряды и применив соотношения (8)- (10), получаем операторное разложение полного взаимодействия в представлении $\left\langle\mathbf{r}_{i}\right|$ :

$$
\begin{aligned}
V\left(\mathbf{r}_{i}\right)= & \sum_{L} \sum_{a, b} \sum_{L^{\prime}} \sum_{a^{\prime}, b^{\prime}}\left|Y_{L a b}^{\ell m}\left(\Omega_{i}\right)\right\rangle{ }^{i} V_{a b a^{\prime} b^{\prime}}^{L L^{\prime}}(r)\left\langle Y_{a^{\prime} b^{\prime}}^{\ell m}\left(\Omega_{i}\right)\right| \\
{ }^{i} V_{a b a^{\prime} b^{\prime}}^{L L^{\prime}}(r)= & \delta_{a a^{\prime}} \delta_{b b^{\prime}} V_{i a b}^{L L^{\prime}}(r)+\sum_{k \neq i} \sum_{c, d}\left\langle a b\left|K\left(\gamma_{k i}\right)\right| c d\right\rangle_{L \ell} \times \\
& \times V_{k c d}^{L L^{\prime}}(r)\left\langle c d\left|K\left(\gamma_{i k}\right)\right| a^{\prime} b^{\prime}\right\rangle_{L^{\prime} \ell},
\end{aligned}
$$

где индексы $a, b, c, d$ и $a^{\prime}, b^{\prime}$ принимают все допустимые при данных $L, \ell, \sigma$ и $L^{\prime}$ значения. Те значения $L$, при которых ${ }^{i} V_{a b a^{\prime} b^{\prime}}^{L L^{\prime}}(r)=0$ для всех возможных $i(1,2,3), a, b, a^{\prime}, b^{\prime}, L^{\prime}$ и $r$, объединим в некоторое множество $\mathcal{E}$. Если оно окажется не пустым $(\mathcal{E} \neq \varnothing)$, то подсуммы рядов $(14)$ с $L \notin \mathcal{E}$ и $L \in \mathcal{E}$ в силу полноты базиса (7) и определений (12) и (13) будут соответственно физическими и ложными слагаемыми для функций класса $\mathcal{A}^{\varepsilon}$ :

$$
\begin{aligned}
V_{k}^{u}\left(\mathbf{r}_{k}\right)=\sum_{L \notin \mathcal{E}} V_{k}^{L u}\left(\mathbf{r}_{k}\right), & V_{k}^{L u}\left(\mathbf{r}_{k}\right) \equiv \sum_{L^{\prime}} \sum_{a, b}\left|Y_{L a b}^{\ell m}\left(\Omega_{k}\right)\right\rangle V_{k a b}^{L L^{\prime}}(r)\left\langle Y_{L^{\prime} a b}^{\ell m}\left(\Omega_{k}\right)\right|, \\
V_{k}^{s}\left(\mathbf{r}_{k}\right)=\sum_{L \in \mathcal{E}} V_{k}^{L s}\left(\mathbf{r}_{k}\right), & V_{k}^{L s}\left(\mathbf{r}_{k}\right) \equiv \sum_{L^{\prime}} \sum_{a, b}\left|Y_{L a b}^{\ell m}\left(\Omega_{k}\right)\right\rangle V_{k a b}^{L L^{\prime}}(r)\left\langle Y_{L^{\prime} a b}^{\ell m}\left(\Omega_{k}\right)\right| .
\end{aligned}
$$

Действительно, по определению множества $\mathcal{E}$ сумма трех операторов $V_{k}^{u}$ отображает хотя бы одну базисную гипергармонику (7) в ненулевую, а сумма трех операторов $V_{k}^{s}$ отображает любую базисную гипергармонику в тождественный нуль независимо от номера $i=1,2,3$ набора $\Omega_{i}$ гиперуглов, в котором эта гипергармоника записана. В силу полноты базиса гипергармоник для любой функции $\Psi$ класса $\mathcal{A}^{\varepsilon}$ и во всех трех координатных представлениях выполняются соотношения (12) и (13). 
Итак, если $\mathcal{E} \neq \varnothing$, то ложные слагаемые сушествуют и верны представления (17) и (18).

Теперь докажем обратное утверждение: если ложные слагаемые сушествуют, то имеется непустое множество $\mathcal{E}$ значений гипермомента такое, что верны представления (18).

Физические (если таковые имеются) и ложные слагаемые каждого взаимодействия $V_{k}$ представим операторными рядами типа (14) по его собственному базису (7). Используя эти ряды и формулы (8) и (9), вычислим матричные элементы сумм (2) физических и ложных слагаемых в базисе $(7)$, записанном в каждом из трех координатных представлений. В результате для каждой допустимой четверки фиксированных значений $L, L^{\prime}$ и $a^{\prime}, b^{\prime}$ получим систему равенств (16), в которой $i=1,2,3$, индексы $a, b$ принимают все возможные при данных $L, \ell, \sigma$ значения, а функция ${ }^{i} V_{a b a^{\prime} b^{\prime}}^{L L^{\prime}}(r)$ отлична от нуля хотя бы при некоторых $L^{\prime}, a^{\prime}, b^{\prime}$ и $r$ или тождественно равна нулю при любых $L^{\prime}, a^{\prime}, b^{\prime}$ и $r$ для физических и ложных слагаемых, соответственно. Те значения $L$, при которых ${ }^{i} V_{a b a^{\prime} b^{\prime}}^{L L^{\prime}} \equiv 0$ при всех $i, a, b$, и $r$, объединим в некоторое множество $\mathcal{E}$ и получим соотношения (17) и (18).

Итак, если ложные слагаемые существуют, то они представимы в виде сумм (18), где $\mathcal{E} \neq \varnothing$.

Опишем другой способ построения множества $\mathcal{E}$. По определению (9) $\left\langle a b\left|K\left(\gamma_{i i}\right)\right| a^{\prime} b^{\prime}\right\rangle_{L \ell}=\delta_{a a^{\prime}} \delta_{b b^{\prime}}$, поэтому в обозначениях

$$
X_{k c d a^{\prime} b^{\prime}}^{L L^{\prime}}(r) \equiv V_{k c d}^{L L^{\prime}}(r)\left\langle c d\left|K\left(\gamma_{k i}\right)\right| a^{\prime} b^{\prime}\right\rangle_{L^{\prime} \ell}
$$

для каждого $L$ система однородных уравнений (16) с любыми фиксированными $L^{\prime}, a^{\prime}, b^{\prime}$ и любыми возможными $i, a, b$ принимает вид

$$
X_{i a b a^{\prime} b^{\prime}}^{L L^{\prime}}(r)+\sum_{k \neq i} \sum_{c, d}\left\langle a b\left|K\left(\gamma_{k i}\right)\right| c d\right\rangle_{L \ell} X_{k c d a^{\prime} b^{\prime}}^{L L^{\prime}}(r)=0 .
$$

Матрица $\mathbf{M}^{L}$ полученной системы уравнений (20) имеет конечную размерность, равную утроенному числу гипергармоник (7) с данными квантовыми числами $L, \ell, m, \sigma$. Согласно критерию разрешимости однородной системы линейных уравнений с конечной матрицей [15] система уравнений (20) имеет нетривиальные решения тогда и только тогда, когда ее матрица вырожденна $\left(\operatorname{det} \mathbf{M}^{L}=0\right)$. Следовательно, множество $\mathcal{E}$ состоит из тех значений $L$, при которых рассматриваемая система уравнений (20) имеет вырожденную матрицу, а матричные элементы $V_{k a b}^{L L^{\prime}}$ парных взаимодействий таковы, что подобные им функции (19) удовлетворяют этой системе при всех $L^{\prime}, a^{\prime}, b^{\prime}$ и $r$.

Для класса $\mathcal{A}^{\varepsilon}$ каждый оператор $V^{L u}$ или $V^{L s}$ логично называть частным физическим или ложным слагаемым взаимодействия $V_{k}$, а для сумм (17) или (18) таких операторов представляется разумным использовать термин обшее физическое или обшее ложное слагаемое того же взаимодействия $V_{k}$.

Пусть $\mathcal{A}$ - класс функций, не обладающих определенными значениями $\ell, m, \sigma$. В этом классе полный базис на сфере $\mathcal{S}^{5}$ образуют гипергармоники (7) со всеми возможными индексами $\ell=0,1, \ldots, m=-\ell,-\ell+1, \ldots, \ell$ и $L, a, b$. Поэтому любое ложное для класса $\mathcal{A}$ слагаемое взаимодействия $V_{k}$ будет ложным для всех классов $\mathcal{A}^{\varepsilon}$. 
Выведем и исследуем достаточные условия сушествования ложных для класса $\mathcal{A}$ слагаемых. Парные взаимодействия представим функциональными рядами по их собственным полным на $\mathcal{S}^{5}$ базисам потенциальных гипергармоник [10, 16]:

$$
V_{k}\left(x_{k}\right)=\sum_{L=0,2, \ldots} V_{k}^{L}(r) Y_{L 00}^{00}\left(\Omega_{k}\right), \quad k=1,2,3 .
$$

Используя эти представления и правила (8) и (9), получим функциональное разложение полного взаимодействия в выбранном координатном представлении $\left\langle\mathbf{r}_{i}\right|$ :

$$
\begin{aligned}
V\left(\mathbf{r}_{i}\right) & =\sum_{L=0,2, \ldots} \sum_{a=0}^{\frac{L}{2}}{ }^{i} V_{a}^{L}(r) Y_{L a a}^{00}\left(\Omega_{i}\right), \\
{ }^{i} V_{a}^{L}(r) & \equiv V_{i}^{L}(r) \delta_{a 0}+\sum_{k \neq i}\left\langle a a\left|K\left(\gamma_{k i}\right)\right| 00\right\rangle_{L 0} V_{k}^{L}(r) .
\end{aligned}
$$

Функции $Y_{L a a}^{00}$ линейно независимы по обоим индексам $L$ и $a$. Поэтому в любом из трех координатных представлений ряд (22) не содержит потенциальных мультиполей $V_{1}^{L}$, $V_{2}^{L}, V_{3}^{L}$ с некоторым фиксированным $L$ тогда и только тогда, когда все мультиполи (23) этого ряда с индексами $i=1,2,3$ и $a=0, \ldots, L / 2$ тождественно равны нулю. Это условие равносильно следующей конечной цепочке не зацепляющихся друг с другом систем из трех $(i=1,2,3)$ тождеств по аргументу $r$ :

$$
\mathbf{N}^{L a} \mathbf{V}^{L}(r)=0 \quad \forall r \geqslant 0, \quad a=0, \ldots, \frac{L}{2} .
$$

Здесь $\mathbf{V}^{L}$ - столбец $\left(V_{1}^{L}, V_{2}^{L}, V_{3}^{L}\right)^{T}$, а $\mathbf{N}^{L a}$ - матрища с элементами

$$
N_{i i}^{L a} \equiv \delta_{a 0}, \quad N_{k i}^{L a} \equiv\left\langle a a\left|K\left(\gamma_{k i}\right)\right| 00\right\rangle_{L 0}, \quad k, i=1,2,3, \quad k \neq i .
$$

Если найдется хотя бы одно значение индекса $a$, при котором матрица $\mathbf{N}^{L a}$ невырожденна, то всей цепочке тождеств (24) будут удовлетворять только тривиальные мультиполи $\left(V_{i}^{L} \equiv 0, i=1,2,3\right)$.

Исследуем условия, при которых матрица $\mathbf{N}^{L a}$ вырожденна. Заменив ее недиагональные элементы (25) по известным формулам $[9,17]$

$$
\left\langle a a\left|K\left(\gamma_{k i}\right)\right| 00\right\rangle_{L 0}=(-1)^{a}\left\langle a a\left|K\left(\gamma_{i k}\right)\right| 00\right\rangle_{L 0}=C_{L a}\left(\sin 2 \gamma_{k i}\right)^{a} P_{\frac{L}{2}-a}^{\left(a+\frac{1}{2}, a+\frac{1}{2}\right)}\left(\cos 2 \gamma_{k i}\right)
$$

где

$$
C_{L a} \equiv \frac{N_{L a a}}{2^{a}} N_{L 00} P_{\frac{L}{2}}^{\left(\frac{1}{2}, \frac{1}{2}\right)}(-1),
$$

и положив по определению $(k, i) \equiv(1,2),(2,3),(3,1)$, получим равенство

$$
\begin{aligned}
\operatorname{det} \mathbf{N}^{L a}= & \delta_{a 0}-\delta_{a 0} C_{L 0}^{2} \sum_{(k, i)}\left(P_{\frac{L}{2}}^{\left(\frac{1}{2}, \frac{1}{2}\right)}\left(\cos 2 \gamma_{k i}\right)\right)^{2}+ \\
& +\left(1+(-1)^{a}\right) C_{L a}^{3} \prod_{(k, i)}\left(\sin 2 \gamma_{k i}\right)^{a} P_{\frac{L}{2}-a}^{\left(a+\frac{1}{2}, a+\frac{1}{2}\right)}\left(\cos 2 \gamma_{k i}\right)
\end{aligned}
$$


Как следует из этого равенства, матрицы $\mathbf{N}^{00}, \mathbf{N}^{20}, \mathbf{N}^{21}$ и все матрицы $\mathbf{N}^{L a}$ с $L>2$ и нечетными $а$ вырожденны при любых допустимых значениях (11) кинематических углов (10), матрицы $\mathbf{N}^{L a}$ с $L=4,6, \ldots$ и четными $a$ могут быть вырожденными только при определенных значениях кинематических углов, а именно когда хотя бы один из трех кинематических углов является нулем функции $P_{L / 2-a}^{(a+1 / 2, a+1 / 2)}(\cos 2 \gamma)$. Далее, если $L=4 p$, где $p$ - некоторое натуральное число, то при $a=L / 2=2 p$ согласно формуле (27)

$$
\operatorname{det} \mathbf{N}^{4 p, 2 p}=2 C_{4 p, 2 p}^{3} \prod_{(k, i)}\left(\sin 2 \gamma_{k i}\right)^{2 p} \neq 0 .
$$

Остается рассмотреть случай $L=4 p+2, p=1,2, \ldots$. При $a=2 p$ по формуле $(27)$ находим

$$
\operatorname{det} \mathbf{N}^{4 p+2,2 p}=2\left(C_{4 p+2,2 p} P^{2 p+\frac{1}{2}, 2 p+\frac{1}{2}}(1)\right)^{3} \prod_{(k, i)}\left(\sin 2 \gamma_{k i}\right)^{2 p} \cos 2 \gamma_{k i} .
$$

Значит, матрица $\mathbf{N}^{4 p+2 p, 2 p}$ вырожденна тогда и только тогда, когда один из кинематических углов равен $\pi / 4$. Согласно соотношениям (11) два кинематических угла одновременно не могут быть равными $\pi / 4$. Пусть $\gamma_{12}=\pi / 4$, тогда $\gamma_{31}, \gamma_{23} \neq \pi / 4$ и в силу равенств (26) имеем $N_{i j}^{4 p+2,2 p}=0, i, j=1,2, N_{13}^{4 p+2 p, 2 p} \neq 0$. Поэтому система уравнений (24) с $L=4 p+2, a=2 p$ имеет только одно нетривиальное решение:

$$
V_{1}^{4 p+2}(r)=\frac{\sin 4 \gamma_{23}}{\sin 4 \gamma_{31}}\left(\frac{\cos 2 \gamma_{23}}{\cos 2 \gamma_{31}}\right)^{2 p-1} V_{2}^{4 p+2}(r), \quad V_{3}^{4 p+2}(r)=0 \quad \forall r,
$$

но это решение не удовлетворяет первой $(L=4 p+2, a=0)$ системе уравнений $(24)$ рассматриваемой цепочки с $L=4 p+2$ ни при каких значениях углов $\gamma_{23}, \gamma_{31}$.

Итак, одновременно нетривиальные мультиполи $V_{i}^{L}, i=1,2,3$, рядов (21) могут удовлетворять тождествам (24) только в двух случаях $L=0, a=0$ и $L=2, a=0,1$. Матрицы этих тождеств вырожденны всегда. Следовательно, для класса $\mathcal{A}$ множество $\mathcal{E}$ может содержать только элементы $L=0$ и $L=2$. Если оно непустое, то для класса $\mathcal{A}$ подсуммы

$$
V_{k}^{u}\left(\mathbf{r}_{k}\right)=\sum_{L \notin \mathcal{E}} V_{k}^{L}(r) Y_{L 00}^{00}\left(\Omega_{k}\right), \quad V_{k}^{s}\left(\mathbf{r}_{k}\right)=\sum_{L \in \mathcal{E}} V_{k}^{L}(r) Y_{L 00}^{00}\left(\Omega_{k}\right)
$$

рядов (21) будут физическими и ложными слагаемыми.

\section{4. УРАВНЕНИЕ ШРЕДИНГЕРА}

Анализ уравнения Шредингера (4) и его общего решения $\Psi \in \mathcal{A}^{\varepsilon}$ в случае $\mathcal{E} \neq \varnothing$ начнем с определения проекционных операторов.

Единичный на $\mathcal{S}^{5}$ оператор $I$ представим в виде суммы $I=P_{u}+P_{s}$ двух ортогональных проекторов

$$
P_{u} \equiv \sum_{L \notin \mathcal{E}} \sum_{a, b}\left|Y_{L a b}^{\ell m}\left(\Omega_{i}\right)\right\rangle\left\langle Y_{L a b}^{\ell m}\left(\Omega_{i}\right)\left|, \quad P_{s} \equiv \sum_{L \in \mathcal{E}} \sum_{a, b}\right| Y_{L a b}^{\ell m}\left(\Omega_{i}\right)\right\rangle\left\langle Y_{L a b}^{\ell m}\left(\Omega_{i}\right)\right| .
$$


Так как преобразование (8) унитарно и сохраняет гипермомент, то представления (29) инвариантны относительно выбора индекса $i=1,2,3$. Поэтому ряды (17) и (18) можно записать в более компактной форме:

$$
V_{k}^{u}=P_{u} V_{k}, \quad V_{k}^{s}=P_{s} V_{k}, \quad k=1,2,3,
$$

где проекторы понимаются как суммы (29), записанные в том же координатном представлении $\left\langle\mathbf{r}_{k}\right|$, что и взаимодействие $V_{k}$. Из соотношений (2) и (30) следует цепочка равенств

$$
\sum_{k=1}^{3} V_{k}^{s}=\sum_{k=1}^{3} P_{s} V_{k}=P_{s} V \equiv 0
$$

справедливая в любом из трех координатных представлений благодаря упомянутой выше инвариантности проекторов. Так как $V$ и $P_{s}$ - эрмитовы операторы, то из тождества $P_{s} V \equiv 0$ следует, что $V P_{s} \equiv 0$. Значит, $\left[P_{s}, V\right]_{-}=0$, а так как $P_{u}=I-P_{s}$, то $\left[P_{u}, V\right]_{-}=0$. Следовательно, оба проектора $P_{s}$ и $P_{u}$ коммутируют с полным гамильтонианом $H \equiv H_{0}-V$. Поэтому собственные числа $p_{u}$ оператора $P_{u}$ являются сохраняюшимися квантовыми числами. Любая функция $U$ из подпространства $\mathcal{U}^{\varepsilon} \equiv P_{u} \mathcal{A}^{\varepsilon}$ является собственной для оператора $P_{u}$ и отвечает его собственному значению $p_{u}=1$. Любая функция $S$ из ортогонального дополнения $\mathcal{S}^{\varepsilon} \equiv P_{s} \mathcal{A}^{\varepsilon}$ подпространства $\mathcal{U}^{\varepsilon}$ до пространства $\mathcal{A}^{\varepsilon}$ принадлежит ядру оператора $P_{u}$, т.е. отвечает собственному значению $p_{u}=0$.

Поясним, в каком смысле пространства $\mathcal{U}^{\varepsilon}$ и $\mathcal{S}^{\varepsilon}$ ортогональны. По определению (29) проекторов $P_{u}$ и $P_{s}$ полный на $\mathcal{S}^{5}$ базис пространства $\mathcal{U}^{\varepsilon}$ или $\mathcal{S}^{\varepsilon}$ образуют гипергармоники $(7)$ с $L \notin \mathcal{E}$ или $L \in \mathcal{E}$. Поэтому эти пространства ортогональны друг другу, вообше говоря, только относительно интегрирования на сфере $\mathcal{S}^{5}$, но по любой из трех $(i=1,2,3)$ совокупностей гиперуглов $\Omega_{i}$.

В уравнение Шредингера (4) подставим искомое в классе $\mathcal{A}^{\varepsilon}$ решение $\Psi$ в виде суммы $\Psi=U+S$ его ортогональных проекций $U \equiv P_{u} \Psi$ и $S \equiv P_{s} \Psi$ на подпространства $\mathcal{U}^{\varepsilon}$ и $\mathcal{S}^{\varepsilon}$. Используя равенства (29)- (31), спроецируем полученное уравнение на эти подпространства. Таким образом, в подпространстве $\mathcal{S}^{\varepsilon}$ получим свободное уравнение Шредингера

$$
\left(H_{0}-E\right) S=0,
$$

а в подпространстве $\mathcal{U}^{\varepsilon}$ - уравнение для физической волновой функции

$$
\left(H_{0}-E\right) U=-\left(P_{u} V\right) U \text {. }
$$

Оно содержит физические слагаемые взаимодействий и определяет энергетический спектр трехчастичной системы. 


\section{5. УРАВНЕНИЯ ФАДДЕЕВА}

Анализ системы уравнений Фаддеева (5) и ее обшего решения $\left(\Psi_{1}, \Psi_{2}, \Psi_{3}\right)$, принадлежашего классу $\mathcal{A}^{\varepsilon}$ в случае $\mathcal{E} \neq \varnothing$, начнем с исследования характерных свойств проекций $U_{k}$ и $S_{k}$ компонент такого решения на подпространства $\mathcal{U}^{\varepsilon}$ и $\mathcal{S}^{\varepsilon}$. По определению проекторов (29) имеем

$$
\Psi_{k}=U_{k}+S_{k}, \quad U_{k} \equiv P_{u} \Psi_{k}, \quad S_{k} \equiv P_{s} \Psi_{k}, \quad k=1,2,3 .
$$

Напомним, что в теории Фаддеева каждая компонента $\Psi_{i}$ и уравнение системы (5), содержашее эту компоненту в своей левой части, записываются в их собственном представлении $\left\langle\mathbf{r}_{i}\right|$.

Докажем, что если все три проекции $U_{1}, U_{2}, U_{3}$ не равны тождественно нулю, то их сумма обладает аналогичным свойством:

$$
\left\langle\mathbf{r}_{i} \mid \sum_{k=1}^{3} U_{k}\left(\mathbf{r}_{k}\right)\right\rangle \neq 0, \quad i=1,2,3
$$

Предположим противное: пусть в пространстве $\mathcal{R}^{6}$ верны три тождества

$$
\left\langle\mathbf{r}_{i} \mid \sum_{k=1}^{3} U_{k}\left(\mathbf{r}_{k}\right)\right\rangle \equiv 0, \quad i=1,2,3 .
$$

Заменим в этих тождествах все слагаемые рядами

$$
U_{k}\left(\mathbf{r}_{k}\right)=\sum_{L \notin \mathcal{E}} \sum_{a, b} U_{k L a b}(r) Y_{L a b}^{\ell m}\left(\Omega_{k}\right)
$$

по их собственным полным угловым базисам пространства $\mathcal{U}^{\varepsilon}$. Спроецировав полученные тождества на такие базисы по правилам (8) и (9), для каждого фиксированного $L \notin \mathcal{E}$ имеем однородную систему тождеств по переменной $r$ :

$$
U_{i L a b}(r)+\sum_{k \neq i} \sum_{c, d}\left\langle a b\left|K\left(\gamma_{k i}\right)\right| c d\right\rangle_{L \ell} U_{k L c d}(r) \equiv 0,
$$

где $i=1,2,3$, а индексы $a, b$ и $c, d$ пробегают все допустимые при данных $L, \ell$ и $\sigma$ значения. Матрица системы (36) совпадает с матрицей $\mathbf{M}^{L}$ системы $(20)$, отвечающей тому же значению $L$. По определению множества $\mathcal{E}$ такая матрица невырожденна. Согласно аль тернативе Фредгольма [15] рассматриваемая однородная система (36) с невырожденной матрищей либо несовместна, либо имеет лишь тривиальное решение. Полученное противоречие означает, что одновременно нетривиальные слагаемые $U_{1}, U_{2}, U_{3}$ фаддеевских компонент (33) всегда обладают свойством (34).

Теперь докажем, что слагаемые $S_{1}, S_{2}, S_{3}$ этих же фаддеевских компонент (33) всегда удовлетворяют во всем пространстве $\mathcal{R}^{6}$ трем тождествам

$$
\left\langle\mathbf{r}_{i} \mid \sum_{k=1}^{3} S_{k}\left(\mathbf{r}_{k}\right)\right\rangle \equiv 0, \quad i=1,2,3 .
$$


В уравнениях (5) заменим все искомые функции суммами (33). Спроецировав полученные уравнения с помошью равенств (29)- (31) на подпространство $\mathcal{S}^{\varepsilon}$, получаем следующие уравнения:

$$
\left(H_{0}-E\right) S_{i}=-P_{s} V_{i} \sum_{k=1}^{3}\left(U_{k}+S_{k}\right), \quad i=1,2,3 .
$$

Опишем решения соответствуюших однородных уравнений

$$
\left(H_{0}-E\right) \widetilde{S}_{i}=0, \quad i=1,2,3 .
$$

Как известно $[11,12]$, при $E<0$ в классе $\mathcal{A}^{\varepsilon}$ имеются только тривиальные решения, если же $E>0$, то любое решение есть ряд

$$
\widetilde{S}_{i}\left(\mathbf{r}_{i}\right)=\sum_{L \in \mathcal{E}} C^{L} \sum_{a, b} D_{i a b}^{L} J_{L+2}(r \sqrt{E}) Y_{L a b}^{\ell m}\left(\Omega_{i}\right), \quad i=1,2,3
$$

содержаший регулярные функции Бесселя $J_{\nu}$, произвольные константы $C^{L}$ и некоторые коэффициенты $D_{i a b}^{L}$. Следовательно, при $E<0$ во всем координатном пространстве $\mathcal{R}^{6}$ выполняются тождества

$$
\left\langle\mathbf{r}_{i} \mid \sum_{k=1}^{3} \widetilde{S}_{k}\left(\mathbf{r}_{k}\right)\right\rangle \equiv 0, \quad i=1,2,3
$$

Докажем эти тождества в случае $E>0$. Для этого заменим функции $\widetilde{S}_{1}, \widetilde{S}_{2}, \widetilde{S}_{3}$ соответствуюшими суммами (40). Применив правила (8) и (9), спроецируем полученные соотношения на их собственные угловые базисы пространства $\mathcal{S}^{\varepsilon}$. Тогда для каждого $L \in \mathcal{E}$ приходим к конечной и по предположению однородной системе уравнений

$$
D_{i a b}^{L}+\sum_{k \neq i} \sum_{c, d}\left\langle a b\left|K\left(\gamma_{k i}\right)\right| c d\right\rangle_{L \ell} D_{k c d}^{L}=0
$$

где $i=1,2,3$, а индексы $a, b, c, d$ принимают все возможные при данных $L, \ell$ и $\sigma$ значения. Матрица этой системы совпадает с вырожденной по определению множества $\mathcal{E}$ матрицей $\mathbf{M}^{L}$ системы уравнений $(20)$, отвечающей тому же значению индекса $L$. Следовательно, если наше предположение о справедливости соотношений (41) верно, то сушествуют коэффициенты $D_{i a b}^{L}$, одновременно не равные нулю. В противном случае, когда правая часть хотя бы одного из уравнений системы (42) отлична от нуля, коэффициенты $D_{i a b}^{L}$ не существуют, т.е. представление (40) не имеет места. Таким образом, получено противоречие, означаюшее справедливость тож деств (41).

В силу равенств (39) и (41) функции (40) с коэффициентами $D_{i a b}^{L}$, подчиненными уравнениям (42), удовлетворяют уравнениям Фаддеева (5) при $E>0$ и любых взаимодействиях. Однако эти функции согласно тождествам (41) отвечают тривиальному решению уравнения Шредингера

$$
\Psi=\widetilde{\Psi} \equiv \sum_{k=1}^{3} \widetilde{S}_{k} \equiv 0
$$


Нетривиальное решение системы уравнений Фаддеева, отвечаюшее тривиальному решению соответствуюшего уравнения Шредингера, принято называть ложным решением. Ссылки на работы, посвяшенные проблеме ложных решений, даны в обзоре [9]. Критерий сушествования таких решений в классе $\mathcal{A}^{\varepsilon}$ доказан в работе [18]. Как было показано, общее решение $\left(S_{1}, S_{2}, S_{3}\right)$ системы уравнений $(38)$,

$$
S_{i}=-\left(H_{0}-E\right)^{-1} P_{s} V_{i} \sum_{k=1}^{3} U_{k}+\widetilde{S}_{i}, \quad i=1,2,3,
$$

содержит ложное решение $\left(\widetilde{S}_{1}, \widetilde{S}_{2}, \widetilde{S}_{3}\right)$, заданное формулами (40) и (42).

Суммируя три равенства (43), записанные в их собственных координатных представлениях, и учитьвая формулы (31) и (41), завершаем доказательство тождеств (37).

Используя представления (33) и тождества (43), спроецируем уравнения (5) на подпространство $\mathcal{U}^{\varepsilon}$. Тогда получим систему уравнений

$$
\left(H_{0}-E\right) U_{i}=-P_{u} V_{i} \Psi=-P_{u} V_{i} \sum_{k=1}^{3} U_{k}, \quad i=1,2,3,
$$

не содержащую ни ложных слагаемых парных взаимодействий, ни проекций фаддеевских компонент (33) на подпространство $\mathcal{S}^{\varepsilon}$. Все решения $\left(U_{1}, U_{2}, U_{3}\right)$ этой системы принадлежат пространству $\mathcal{U}^{\varepsilon}$ и поэтому обладают дополнительным к набору $\varepsilon$ квантовым числом $p_{u}=1$. В силу соотношений $(33),(34)$ и (37) каждому нетривиальному решению $\left(U_{1}, U_{2}, U_{3}\right)$ отвечает нетривиальное решение $(3)$ уравнения Шредингера $(32)$

$$
U=\sum_{k=1}^{3} U_{k}
$$

не содержашее проекций $S_{k}, k=1,2,3$

Для полноты рассмотрим особьй случай, когда $\mathcal{E} \neq \varnothing$, но $U_{1}=U_{2}=U_{3} \equiv 0$. В этом случае согласно формулам (33) и (43) получаем $\Psi_{k}=S_{k}=\widetilde{S}_{k}, k=1,2,3$. Поэтому исходные (стандартные) уравнения Фаддеева (5) имеют только ложные решения (40), (42), а соответствуюшая им волновая функция (45) является тривиальной. Модифицированные уравнения Фаддеева (44) по их построению не имеют ложных решений.

Как было показано, ложные слагаемые парных взаимодействий порождают ложные и, вообще говоря, нетривиальные слагаемые (43) фаддеевских компонент (33). Приведем схему доказательства обратного утверждения: если фаддеевские компоненты имеют ложные слагаемые, то и парные взаимодействия содержат ложные слагаемые.

Пусть верны представления (33), неравенства (34) и тождества (37). Спроецируем эти тождества на полный угловой базис (7) пространства $\mathcal{A}^{\varepsilon}$. Применив альтернативу $\Phi$ редгольма, докажем, что сушествует некоторое множество $\mathcal{E}$ таких значений $L$, что цепочки полученных неоднородных или однородных систем уравнений для гиперсферических компонент $U_{k L a b}$ или $S_{k L a b}$ слагаемых $U_{k}$ или $S_{k}, k=1,2,3$, имеют нетривиальные решения, если $L \notin \mathcal{E}$ или $L \in \mathcal{E}$, соответственно. Введем проекторы (29), подействуем 
проектором $P_{s}$ на уравнения (5) и учтем неравенства (34) и тождества (37). Таким образом получим три выполняюшихся равенства (43). Суммируя их и введя обозначения $V_{k}^{s} \equiv P_{s} V_{k}, k=1,2,3$, приходим к тождествам (13), означаюшим, что ложные слагаемые парных взаимодействий существуют.

Обсудим формулировки (4), (5) и (44) задачи трех частиц с точки зрения закона сохранения квантового числа $p_{u}=1$.

Согласно представлению (33) решения стандартных уравнений Фаддеева (5), вообше говоря, не обладают квантовым числом $p_{u}=1$. Действительно, $P_{u} \Psi_{k}=U_{k} \neq \Psi_{k}$, $k=1,2,3$. Однако этим числом обладают физические слагаемые $\left(U_{1}, U_{2}, U_{3}\right)$ таких решений и их сумма (45), т.е. волновая функция $U$, удовлетворяющая уравнению Шредингера (4). Физические слагаемые $U_{1}, U_{2}, U_{3}$ подчинены модифицированным уравнениям Фаддеева (44). Эти уравнения, как и уравнение Шредингера (4), содержат лишш физические слагаемые парных взаимодействий. Поэтому эквивалентность модифицированных уравнений Фаддеева и уравнения Шредингера не вызывает сомнений. В силу этой эквивалентности и представления (45) исходная система уравнений Фаддеева (5) также эквивалентна уравнению Шредингера (4), несмотря на то что эта система содержит ложные слагаемые и взаимодействий, и фаддеевских компонент.

\section{6. ЗАМЕЧАНИЯ И ПРИМЕРЫ}

Докажем, что в случае трех тождественных бозонов для любого класса $\mathcal{A}^{\varepsilon}$ проекторы $P_{u}$ и $P_{s}$, заданные равенствами (29), вырождаются соответственно в оператор симметризации $S^{+}$и оператор $I-S^{+}$. В рассматриваемом случае [11]

$$
3 S^{+} Y_{L a b}^{\ell m}\left(\Omega_{i}\right)=\sum_{c, d} A_{c d a b}^{L \ell} Y_{L c d}^{\ell m}\left(\Omega_{i}\right)
$$

где

$$
A_{c d a b}^{L \ell} \equiv \delta_{a c} \delta_{b d}+\left(1+(-1)^{b+d}\right)\left\langle a b\left|K\left(\frac{\pi}{3}\right)\right| c d\right\rangle_{L \ell},
$$

а условия (20), определяюшие ложные слагаемые, принимают вид равенств

$$
\sum_{c, d} A_{c d a b}^{L \ell} X_{k c d a^{\prime} b^{\prime}}^{L L^{\prime}}=0, \quad k=1,2,3,
$$

содержаших те же самые коэффициенты $A_{c d a b}^{L \ell}$. Если $Y_{L a b}^{\ell m}$ - симметричная по всем перестановкам функция, то согласно формулам (46) все коэффициенты $A_{c d a b}^{L \ell}$, за исключением $A_{a b a b}^{L \ell}=3$, равны нулю, и поэтому условия (47) не выполняются. Если $Y_{L a b}^{\ell m}-$ функция любой другой симметрии, то из формул (46) следует, что все коэффишиенты $A_{c d a b}^{L \ell}$ равны нулю и условия (47) становятся тождествами для любых $X_{k c d a^{\prime} b^{\prime}}^{L L^{\prime}}$. Значит, множество $\mathcal{E}$ состоит только из значений индексов $L$ гипергармоник $Y_{L a b}^{\ell m}$, не обладаюших полной перестановочной симметрией. Поэтому равенства (29), определяюшие проекторы $P_{u}$ и $P_{s}$, становятся спектральными представлениями соответствуюших операторов $S^{+}$и $I-S^{+}$, что и требовалось доказать. 
Предложенный способ построения ложных слагаемых поясним на примере подобных центральных взаимодействий

$$
V_{k}\left(x_{k}\right)=c_{k} F\left(x_{k}\right), \quad k=1,2,3,
$$

где $c_{k}$ и $F$ - некоторые ненулевые коэффициенты и функция. Условия (24), определяюшие частные ложные слагаемые ( $L$ фиксировано) таких взаимодействий для класса функций $\mathcal{A}$, сводятся к совокупности условий для столбца $\mathbf{C}$ :

$$
\mathbf{N}^{L a} \mathbf{C}=0, \quad \mathbf{C} \equiv\left(c_{1}, c_{2}, c_{3}\right)^{T}, \quad a=0,1, \ldots, \frac{L}{2} .
$$

Пусть $L=0$. Тогда индекс $a$ принимает только одно значение $a=0$. Согласно формулам (26) все элементы (25) матрицы $\mathbf{N}^{00}$ равны единице независимо от значений кинематических углов, и рассматриваемая система условий (49) вырождается в требование

$$
c_{1}+c_{2}+c_{3}=0
$$

Если оно выполняется, то согласно определению (28) слагаемые

$$
V_{k}^{L s}\left(\mathbf{r}_{k}\right)=c_{k} Y_{L 00}^{00}\left(\Omega_{k}\right) F^{L}(r)=\left(\delta_{L 0}+L \delta_{L 2}\right) \pi^{-\frac{3}{2}} F^{L}(r) \cos L \varphi_{k},
$$

где

$$
F^{L}(r) \equiv(4-L) \pi^{-\frac{1}{2}} \int_{0}^{\frac{\pi}{2}} d \varphi(\sin (L+2) \varphi)^{2} F(r \cos \varphi),
$$

подобных парных взаимодействий (48) будут частными ложными слагаемыми с $L=0$ для класса функций $\mathcal{A}$.

Пусть $L=2$, тогда имеются две $(a=0,1)$ системы условий (49). В силу соотношений (11) и (25)- (27)

$$
N_{k i}^{2 a}=\delta_{k i}(-1)^{a} \cos \left(2 \gamma_{k i}-a \frac{\pi}{2}\right), \quad \operatorname{det} \mathbf{N}^{2 a}=0, \quad a=0,1, \quad k, i=1,2,3,
$$

поэтому обе исследуемые системы матричных условий (49) сводятся к одной и той же совокупности двух условий:

$$
c_{1} \sin 2 \gamma_{12}=c_{3} \sin 2 \gamma_{23}, \quad c_{2} \sin 2 \gamma_{12}=c_{3} \sin 2 \gamma_{31} .
$$

Если коэффициенты $c_{1}, c_{2}, c_{3}$ подчиняются этим условиям, то взаимодействия (48) имеют частные $(L=2)$ ложные для класса функций $\mathcal{A}$ слагаемые, заданные формулами $(51)$ c $L=2$.

Заметим, что условия (50) и (52) несовместны, поэтому в случае подобных парных взаимодействий множество $\mathcal{E}$ для класса $\mathcal{A}$ состоит всего из одного элемента: $L=0$ или $L=2$.

Для любых тождественных бозонов парные взаимодействия являются подобными, в формулах (48) $c_{1}=c_{2}=c_{3}=c \neq 0$, а по определению (10) $\gamma_{12}=\gamma_{31}=\gamma_{23}=\pi / 3$. 
Поэтому соотношения (50) неверны, а условия (52) заведомо выполняются. Значит, в случае трех тождественных бозонов $\mathcal{E}=\{L=2\}$, а физические и ложные для класса $\mathcal{A}$ слагаемые (28) принимают вид

$$
V_{k}^{u}\left(\mathbf{r}_{k}\right)=c \sum_{L \neq 2} F^{L}(r) Y_{L 00}^{00}\left(\Omega_{k}\right), \quad V_{k}^{s}=c F^{2}(r) Y_{200}^{00}\left(\Omega_{k}\right) .
$$

Пусть взаимодействия (48) кулоновские:

$$
F\left(x_{k}\right)=\frac{1}{x_{k}}, \quad c_{k}=\hbar^{-1} z_{i} z_{j} e^{2} \sqrt{2 \mu_{i j}}, \quad k=1,2,3,
$$

где $\mu_{i j}$ - приведенная масса, а $z_{i} e$ и $z_{j} e-$ заряды частиц с номерами $i$ и $j$. Тогда достаточные условия (50) и (52) существования частных ложных слагаемых с $L=0$ и $L=2$ сводятся к соответствующим условиям

$$
\begin{gathered}
z_{1} z_{2} \sqrt{\frac{m_{1} m_{2}}{m_{1}+m_{2}}}+z_{2} z_{3} \sqrt{\frac{m_{2} m_{3}}{m_{2}+m_{3}}}+z_{1} z_{3} \sqrt{\frac{m_{1} m_{3}}{m_{1}+m_{3}}}=0 \\
\left(z_{1} \delta_{i 2}+z_{2} \delta_{i 1}\right)\left(\frac{m_{i}+m_{3}}{m_{1}+m_{2}}\right)^{\frac{3}{2}}=z_{3}\left(\frac{m_{3}}{m_{1} \delta_{i 2}+m_{2} \delta_{i 1}}\right)^{\frac{1}{2}}, \quad i=1,2,
\end{gathered}
$$

а формулы (51), описывающие такие слагаемые, принимают вид

$$
V_{k}^{L s}\left(\mathbf{r}_{k}\right)=\hbar^{-1} z_{i} z_{j} e^{2} \sqrt{2 \mu_{i j}}\left(1-\frac{L}{10}\right) \frac{16}{3 \pi^{2} r} \cos L \varphi_{k}, \quad L=0,2 .
$$

Если $z_{1}=z_{2}=-z_{3}$, то соотношение (53) выполняется для частиц с массами $m_{1}=$ $m_{2}=7 m_{3}$. С относительной точностью $10^{-3}$ такие массы имеют системы, в которых одна частица - антипротон (протон) $\left(m_{3} \approx 1.007\right.$ а.е.м.), а две другие - положительно (отрицательно) заряженные ионы атома ${ }^{7} \mathrm{Li}\left(m_{1}=m_{2} \approx 7.02\right.$ а.е.м. [19]).

В случае $z_{1}=z_{2} / 2=z_{3}=1$ оба условия (54) выполняются при $m_{1}=m_{3}=$ 1 а.е.м., $m_{2} \approx 4.09777$ а.е.м. или при $m_{1}=m_{3}=2$ а.е.м., $m_{2} \approx 8.19548$ а.е.м. Близкие к указанным значениям массы имеют частицы следуюших систем: два протона $\left(m_{1}=m_{3}\right)$ и двухзарядный ион ${ }^{4} \mathrm{He}^{++} \quad\left(m_{2} \approx 4.0026\right.$ а.е.м. $)$ или два дейтрона $\left(m_{1}=m_{3} \approx 2.0141\right.$ а.е.м. $)$ и двухзарядный ион ${ }^{8} \mathrm{Be}^{++} \quad\left(m_{2} \approx 8.005\right.$ а.е.м. $)$ [19].

Обсудим физическую роль ложных слагаемых парных взаимодействий.

Пусть $A=\left(V_{1}, V_{2}, V_{3}\right)$ и $\tilde{A}=\left(\widetilde{V}_{1}, \widetilde{V}_{2}, \widetilde{V}_{3}\right)$ - два набора парных взаимодействий, одинаково хорошо описывающих все имеющиеся данные по рассеянию и спектрам в каждой паре некоторых частищ $a, b, c$. Из таких фазово-эквивалентных наборов принято считать реалистическим тот, используя который удается лучше описать всю имеющуюся совокупность данных о свойствах трехчастичной системы $a b c$. Если оба набора $A$ и $\tilde{A}$ не имеют ложных слагаемых, то упомянутый общепринятый рецепт представляется непротиворечивым.

Пусть набор $A$ имеет ложные слагаемые, а набор $\tilde{A}$ не обладает таковыми. Если для вычислений по уравнениям Шредингера или Фаддеева используется набор $\tilde{A}$, то исследуемые свойства системы $a b c$ определяются всеми взаимодействиями $\widetilde{V}_{1}, \widetilde{V}_{2}, \widetilde{V}_{3}$. Если 
же для аналогичных вычислений используется набор $A$, то свойства той же системы $a b c$ определяются не всеми взаимодействиями $V_{1}, V_{2}, V_{3}$, а лишь их физическими слагаемыми $V_{1}^{u}, V_{2}^{u}, V_{3}^{u}$. В рассмотренном случае общепринятый рецепт выбора реалистического набора оказывается некорректным по двум причинам. Во-первых, если взаимодействия в системе $a b c$ описываются набором $A(\tilde{A})$, то все ее состояния обладают (не обладают) квантовым числом $p_{u}=1$. Во-вторых, набору $\tilde{A}$ сопоставляется не набор $A$, а набор $A^{u} \equiv\left(V_{1}^{u}, V_{2}^{u}, V_{3}^{u}\right)$. Следовательно, для корректного выбора реалистического набора из наборов $A$ и $\tilde{A}$ сравнительным анализом трехчастичных данных необходимо, чтобы оба набора либо не имели ложных слагаемых, либо имели ложные слагаемые, определенные одним и тем же множеством $\mathcal{E}$.

Проблема выбора реалистических парных взаимодействий часто возникает в ядерной физике низких энергий. В пределе низких энергий хорошим приближением считается замена сильных взаимодействий их $S$-волновыми компонентами. Для класса функций $\mathcal{A}^{\varepsilon}$ такие компоненты - суммы (14), в которых $a=\ell$ и $b=0$. Поэтому условия, определяющие ложные слагаемые $S$-волновых взаимодействий, суть однородные уравнения (16), в которых $V_{i a b}^{L L^{\prime}} \equiv 0$ при всех $\mathbf{r}_{i}, i=1,2,3$ и $a \neq \ell, b \neq 0$.

Как следует из представлений (17) и (28), физические слагаемые парных взаимодействий описьвают трехчастичные силы. Например, согласно формулам (1) и (55) в случае $\mathcal{E}=\{L=0\}$ физические слагаемые кулоновских взаимодействий зависят и от координаты $y_{k}$ третьей частицы:

$$
V_{k}^{u}=V_{k}-V_{k}^{s}=\hbar^{-1} z_{i} z_{j} e^{2} \sqrt{2 \mu_{i j}}\left(\frac{1}{x_{k}}-\frac{16}{3 \pi^{2}} \frac{1}{\sqrt{x_{k}^{2}+y_{k}^{2}}}\right)
$$

Значит, определенные трехчастичные силы могут появляться естественным образом, а именно как эффект исключения ложных слагаемых двухчастичных сил из полного взаимодействия.

Столь простая причина возникновения трехчастичных сил ранее не обсуждалась [20]. Наиболее интересным представляется сравнение строений трехчастичного полного взаимодействия в случае ложных слагаемых и в случаях, исследованных в работах [21-23]. В этих работах было показано, что трехчастичные силы в системах из трех составных частиц (кластеров) $a, b$ и $c$ появляются как эффект исключения определенных компонент в двухчастичных взаимодействиях. В результате полное взаимодействие $V_{a b c}$ в системе трех таких частищ представляется суммой трех парных взаимодействий $V_{a b}$, $V_{a c}, V_{b c}$ и трехчастичного взаимодействия $V_{a b c}^{(3)}$ :

$$
V_{a b c}=V_{a b}+V_{a c}+V_{b c}+V_{a b c}^{(3)}
$$

Согласно основным выводам работ $[21,22]$ вклад трехчастичного взаимодействия $V_{a b c}^{(3)}$, обусловленного принципом Паули или короткодействующими корреляциями, или поляризацией кластеров, в полное взаимодействие $V_{a b c}$ мал. 
Совершенно иное строение имеет полное взаимодействие $V$, если существуют ложные слагаемые двухчастичных взаимодействий. В этом случае согласно представлениям (1) и (17) полное взаимодействие становится полностью трехчастичным, т.е. не содержит никаких двухчастичных компонент.

Ложные слагаемые перенормируют константы исходных парных взаимодействий в определенных трехчастичных конфигурациях. Например, в случае, описываемом формулой $(56)$, имеем $V_{k}^{u} / V_{k} \approx 0.4$ для всех $x_{k}$ и $y_{k}=0$. Значит, кулоновский барьер в паре одноименно заряженных частиш, имеющих номера $i$ и $j$ и лежаших на одной прямой с третьей частицей $\left(y_{k}=0\right)$, оказывается более низким по сравнению с барьером в этой же паре в отсутствие третьей частицы $\left(y_{k}=\infty\right)$. Таким образом, можно ожидать, что вероятность слияния двух одноименно заряженных ядер может существенно увеличиться в присутствии такой третьей частицы, для которой кулоновские взаимодействия имеют ложные слагаемые с $L=0$.

\section{7. ЗАКЛЮЧЕНИЕ}

Перечислим основные результаты настоящей работы.

Ложные для класса $\mathcal{A}^{\varepsilon}$ слагаемые центральных парных взаимодействий существуют тогда и только тогда, когда некоторые коэффищиенты гиперсферического разложения полного взаимодействия тож дественно равны нулю. Эти условия имеют вид конечных цепочек однородных тождеств (16) для коэффициентов гиперсферических разложений парных взаимодействий (21).

Факт существования всех ложных слагаемых парных взаимодействий для класса функций $\mathcal{A}^{\varepsilon}$ эквивалентен закону сохранения квантового числа $p_{u}=1$. Ложные слагаемые фаддеевских компонент волновой функции, подчиненные стандартным уравнениям Фаддеева (5), сушествуют тогда и только тогда, когда сушествуют ложные слагаемые парных взаимодействий. Ложные слагаемые не нарушают эквивалентность уравнения Шредингера (4) и уравнений Фаддеева (5).

\section{Список литературы}

[1] Л. Д. Ландау, Е. М. Лифииц. Квантовая механика. М.: Наука, 1974.

[2] С. П. Меркурьев, Л. Д. Фаддеев. Квантовая теория рассеяния для систем нескольких частиц. М.: Наука, 1985.

[3] Gy. Bencze. Nucl. Phys. A. 1973. V. 210. P. 568.

[4] E.F. Redish. Nucl. Phys. A. 1974. V. 225. P. 16.

[5] V.I. Kukulin, V. N. Pomerantsev. Ann. Phys. 1978. V. 111. P. 333.

[6] В.И. Кукулин, В. Н. Померанцев. ЯФ. 1978. Т. 27. С. 1668.

[7] J.L.Friar, B.F. Gibson, G.L. Payne. Phys. Rev. C. 1980. V. 22. P. 284.

[8] M. Fabre de la Ripelle, S. Y. Larsen. Few-Body Syst. 1992. V. 13. P. 199.

[9] В. В. Пупьшев. ЭЧАЯ. 1999. Т. 30. С. 1562.

[10] M. Fabre de la Ripelle. The hyperspherical expansion method. In: Lect. Notes Phys. V.273. Models and Methods in Few-Body Physics. Eds. L. S. Ferreire, A. C. Fonseca, L. Streit. Berlin: Springer, 1987. Р. 283.

[11] Р. И. Джибути, Н. Б. Крупенникова. Метод гиперсферических функций в квантовой механике нескольких тел. Тбилиси: Мецниереба, 1984. 
[12] Н. Я. Виленкин. Специальные функции и теория представления групп. М.: Наука, 1965.

[13] В. В. Пупышев. ЯФ. 1999. Т. 62. С. 1955.

[14] J. Raynal, J. Revai. Nuovo Cimento. A. 1970. V. 68. P. 611.

[15] P. Lankaster. Theory of Matrices. New York-London: Academic Press, 1969.

[16] В. Д. Эфрос. ЯФ. 1972. Т. 15. С. 226.

[17] Я. А. Смородинский, В. Д. Эфрос. ЯФ. 1973. Т. 17. С. 211.

[18] В. В. Пупышев. ТМФ. 1996. Т. 117. С. 501.

[19] О. Ф. Немец, Ю. В. Гофман. Справочник по ядерной физике. Киев: Наукова думка, 1975.

[20] The Three-Body Force in the Three-Nucleon System. Lect. Notes Phys. V.260. Eds. B. L. Berman, B. F. Gibson. Berlin: Springer, 1986.

[21] S. Nakaichi-Maeda, E. W. Schmid. Z. Phys. A. 1984. V. 318. P. 287.

[22] E.W. Schmid, S. Nakaichi-Maeda. Three-body forces in the constituent quark model. In: Procced. of the VII International Seminar on Problems in High-Energy Physics. Eds. A. I. Titov, E. K. Aksenova, E. V. Ivashkevich. Dubna: JINR, 1984. P. 429.

[23] W. N. Polyzou, W. Glöckle. Few-Body Syst. 1990. V. 9. P. 97.

Поступила в редакцию 17.XII.1999 г., после доработки 6.VI.2000 г. 